\title{
Encapsulation of Magnetic Nanoparticles with Biopolymer for Biomedical Application
}

\author{
Suk Fun Chin, Mohamed Makha, Colin L Raston \\ School of Biomedical, Biomolecular and Chemical Sciences \\ The University of Western Australia, Crawley, Western Australia 6009 \\ Email: clraston@cyllene.uwa.edu.au \\ Telephone: (618) 64881572, Fax: (618) 64881005
}

\begin{abstract}
Magnetite nanoparticles were synthesized by coprecipitation of $\mathrm{Fe}^{2+}$ and $\mathrm{Fe}^{3+}$ with $\mathrm{NH}_{4} \mathrm{OH}$ using Spinning Disc Processing (SDP). Chitosan was then coated on the surface of magnetite nanoparticles using SDP. FTIR study and zeta potential measurement confirmed the absorption of chitosan unto the surface of magnetite nanoparticles. Transmission electron microscope (TEM) image showed that the particle sizes are in the range $10-200 \mathrm{~nm}$.
\end{abstract}

\section{Keywords-magnetite, nanoparticles; chitosan; coating}

\section{INTRODUCTION}

Magnetic nanoparticles have been extensively studied because of their potential applications as contrast agents in magnetic resonance imaging (MRI) of tumors, cell and DNA separation, magnetically guided drug delivery, tumor hyperthermia etc. [1-3]. Among the magnetic oxides, magnetite nanoparticles are most suitable due to their low toxicity and good magnetic properties. Magnetite is a ferromagnetic iron oxide, $\mathrm{Fe}_{3} \mathrm{O}_{4}$ with an inverse spinel crystalline structure in which part of the iron atoms are octahedrally coordinated to oxygen and the rest are tetrahedrally coordinated to oxygen [4]. However, magnetite tends to aggregate due to strong magnetic dipole-dipole attractions between particles combined with inherently large surface energy.

In order to successfully prepare stable magnetite dispersions, any attractive forces between the nanoparticles must be overcome. Stabilizers or surfactants have been used to prevent sedimentation of these nanoparticles. The choice of stabilizer relates to its ability to interact with magnetite particles via functional groups and form a tightly bonded monomolecular layer around the particles. Another possible way of stabilizing iron oxide nanoparticles in aqueous solution is to encapsulate them with polymeric materials. The polymer coatings serve as a steric barrier which reduces magnetic attractions between the particles. Besides these, coatings on magnetite nanoparticles can also improve chemical stability by protecting the particles surface from oxidation.

In this study, encapsulation of magnetite nanoparticles using chitosan coupled with Spinning Disk Processing (SDP) technique is reported. As a natural biopolymer, chitosan is a hydrophilic, biocompatible, biodegradable and non-toxic polymer, making it attractive for biomedical applications [5-6]. The presence of a shell of this biopolymer around magnetite nanoparticles will not only improve the biocompatibility of magnetite nanoparticles but also provides amino and hydroxyl groups for interplay with biomolecules. Furthermore, chitosan can be produced by deacetylation of chitin. Chitin is the main component of exoskeleton of crustaceans and is the second most abundant natural polymer after cellulose, and therefore is a cheap, renewable biopolymer [7].

\section{MATERIALS AND METHOD}

\section{A) Synthesis of magnetite nanoparticles}

Magnetite nanoparticles were prepared by coprecipitation of ferric and ferrous chlorides (1:2) with aqueous ammonia solution. Both solutions were purged with argon gas for at least 15 minutes and then continuously fed into the SDP at feeding rate of $0.5 \mathrm{ml} / \mathrm{s}$. The disk spinning rate was $2000 \mathrm{rpm}$. The synthesis was carried out under an argon atmosphere. The conical flask contained the resulting magnetite was placed on a permanent magnet, the supernatant solution was discarded. Deoxygenated ultra pure deionized water was added to wash the magnetite nanoparticles. This process was repeated for several times until the $\mathrm{pH}$ of the suspension was nearly neutral. The magnetite nanoparticles were then re-dispersed in deoxygenated deionized water.

\section{B) Coating of magnetite nanoparticles with chitosan}

Chitosan solution was prepared by dissolved various amount of chitosan in $1 \%$ of acetic acid. Chitosan coated magnetite nanoparticles were prepared by mixing the preformed magnetite nanoparticles with chitosan solution using SDP at feeding rate of $0.5 \mathrm{ml} / \mathrm{s}$ and at $1500 \mathrm{rpm}$ disk spinning rate. The chitosan coated magnetite nanoparticles were then washed several times with deionized water to remove the excess chitosan in the suspension.

\section{C) Characterization}

The FTIR spectra of the samples were recorded on a Perkin Elmer FTIR Spectrometer at $4 \mathrm{~cm}^{-1}$ resolution. Samples were ground-blended with $\mathrm{KBr}$ and the compressed to form pellet. All the spectra were recorded at the range of $400-4000 \mathrm{~cm}^{-1}$.

Zeta potentials of uncoated magnetite and chitosan coated magnetite as a function of $\mathrm{pH}$ were determined using a Zetasizer Nano ZS series (Malvern Instruments Ltd., UK). 